\title{
Combination of Acacetin with Antibiotics against Methicillin Resistant Staphylococcus aureus Isolated from Clinical Specimens
}

\author{
Jeong-Dan Cha' ${ }^{1}$, Sung-Mi Choi ${ }^{2}$, Jeong Hye Park ${ }^{3}$ \\ ${ }^{1}$ Department of Research Development, Institute of Jinan Red Ginseng, Jinan, South Korea \\ ${ }^{2}$ Department of Dental Hygiene, Daegu Health College, Daegu, South Korea \\ ${ }^{3}$ Department of Nursing, Dong-eui University, Busan, South Korea \\ Email: ${ }^{*}$ ihpark@deu.ac.kr
}

Received 17 December 2013; revised 25 February 2014; accepted 19 March 2014

Copyright (C) 2014 by authors and Scientific Research Publishing Inc.

This work is licensed under the Creative Commons Attribution International License (CC BY). http://creativecommons.org/licenses/by/4.0/

(c) (i) Open Access

\begin{abstract}
Methicillin-restitant Staphylococcus aureus (MRSA) is very dangerous bacteria and one of the most feared nosocomial germs. In this study, acacetin was evaluated against 20 clinical isolates of MRSA, either alone or in combination with antibiotics. The acacetin exhibited a good activity against isolates MRSA with MICs/MBCs ranged between 10 - 80/20 - $160 \mu \mathrm{g} / \mathrm{mL}$, for ampicillin 64 - 1024/128 $-2048 \mu \mathrm{g} / \mathrm{mL}$, and for oxacillin $8-32 / 16-64 \mu \mathrm{g} / \mathrm{mL}$. The combination of acacetin plus oxacillin or ampicillin was reduced by $\geq 4$-fold against isolates MRSA tested, evidencing a synergistic effect as defined by a FICI of $\leq 0.5$. Furthermore, a time-kill study evaluating the growth of the tested bacteria was completely attenuated after $2-5 \mathrm{~h}$ of treatment with the 1/2 MIC of acacetin, regardless of whether it was administered alone or with oxacillin (1/2 MIC) or ampicillin (1/2 MIC). In conclusion, acacetin exerted synergistic effects when administered with oxacillin or ampicillin and the antibacterial activity and resistant regulation of acacetin against clinical isolates of MRSA might be useful in controlling MRSA infections.
\end{abstract}

\section{Keywords}

Acacetin; Methicillin-Resistant Staphylococcus aureus; Minimum Inhibitory Concentrations; Minimum Bactericidal Concentrations; Time-Kill Curves; Fractional Inhibitory Concentration

\section{Introduction}

Staphylococcus aureus (S. aureus) is an important human pathogen, causing life-threatening systemic infections

"Corresponding author.

How to cite this paper: Cha, J.-D., et al. (2014) Combination of Acacetin with Antibiotics against Methicillin Resistant Staphylococcus aureus Isolated from Clinical Specimens. Advances in Bioscience and Biotechnology, 5, 398-408.

http://dx.doi.org/10.4236/abb.2014.54048 
such as pneumonia, septicemia, endocarditis, and osteomyelitis [1] [2]. Furthermore, S. aureus can spread easily, and have been found in the noses of approximately $40 \%$ - 50\% of healthy people, is the etiological agent more commonly associated to the disease, and is normally related to subclinical or chronic infections [3] [4]. Clinical isolates of methicillin-resistant Staphylococcus aureus (MRSA) have become the most common cause of infections among pathogenic bacteria around the Globe, and many life-threatening diseases such as endocarditis, pneumonia and toxin shock syndrome are ascribed to them [4] [5]. Contrary to methicillin-susceptible $S$. aureus (MSSA), MRSA tend to be multi-drug resistant (MDR), that is, resistant not only to $\beta$-lactam antibiotics but also to a wide range of different antibiotic classes, such as fluoroquinolones, tetracyclines, macrolides, lincosamides, and aminoglycosides, and even strains of vancomycin intermediate susceptible or full resistant (VISA and VRSA, respectively) have emerged [6] [7]. Antimicrobial drugs effective for treatment of patients infected with MRSA are limited. Thus, it is important and valuable to find compounds that potentiate antimicrobial activity of antibiotics.

Many plant-derived medicines used in traditional medicinal systems have been recorded in pharmacopeias as agents used to treat infections and a number of these have been recently investigated for their efficacy against MRSA [8] [9]. Flavonoids have also been shown to exhibit broader bioactivities such as protection of vascular integrity, antihepatotoxicity, anti-inflammatory activity, antitumor effect, antiallergic properties, and antimicrobial effects [9]-[13]. Acacetin (5,7-dihydroxy-4'-methoxyflavone), a flavone compound found in several plants, has been reported to show anti-peroxidative, anti-mutagenic, anti-cancer, anti-inflammatory, antibacterial, and anti-plasmodial activities [14]-[16]. Drug synergism between known antibiotic and bioactive plant extracts is a novel concept and could be benificial (synergistic or addition interaction) or deleterious (antagonistic or toxic outcome) [7] [17]. Although a broad range of biological and pharmacological activities of acacetin have been reported, the mechanism(s) behind its antibacterial effects are not fully understood.

In this study, the antimicrobial activities of acacetin against methicillin-resistant Staphylococcus aureus isolated in a clinic were assessed using broth microdilution method and the checkerboard and time-kill methods for synergistic effect of the combination with ampicillin or oxacillin.

\section{Materials and Methods}

\subsection{Preparation of Bacterial Strains}

20 isolates of methicillin-resistant Staphylococcus aureus isolated from the Wonkwang University Hospital, as well as standard strains of methicillin-sensitive S. aureus (MSSA) ATCC 25923 and methicillin-resistant S. aureus (MRSA) ATCC 33591 were used. Antibiotic susceptibility was determined in testing the inhibition zones (inoculums $0.5 \mathrm{McF}$ arland suspension, $1.5 \times 10^{8} \mathrm{CFU} / \mathrm{ml}$ ) and MIC/MBC (inoculums $5 \times 10^{5} \mathrm{CFU} / \mathrm{ml}$ ) for strains, measured as described in the National Committee for Clinical Laboratory Standards (NCCLS, 1999). To rapidly identifying the methicillin-resistance, presence of mecA gene in MRSA isolates was detected using PCR method as the following [18].

\subsection{Minimum Inhibitory Concentration/Minimum Bactericidal Concentration Assay}

The antimicrobial activities of acacetin against clinical isolates MRSA 20 and reference strains were determined via the broth dilution method [17] [19]. The minimum inhibitory concentration (MIC) was recorded as the lowest concentration of test samples resulting in the complete inhibition of visible growth. For clinical strains, $\mathrm{MIC}_{50} \mathrm{~S}$ and $\mathrm{MIC}_{90} \mathrm{~s}$, defined as MICs at which, 50\% and 90\%, respectively of the isolates were inhibited, were determined. The minimum bactericidal concentration (MBC) was determined based on the lowest concentration of the extracts required to kill 99.9\% of bacteria from the initial inoculum as determined by plating on agar.

\subsection{Checkerboard Dilution Test}

The synergistic combinations were investigated in the preliminary checkerboard method performed using the MRSA, MSSA, and one clinical isolate strains via MIC determination [19] [20]. The fractional inhibitory concentration index (FICI) is the sum of the FICs of each of the drugs, which were defined as the MIC of each drug when used in combination divided by the MIC of each drug when used alone. The FIC index was calculated as follows: $\mathrm{FIC}=$ (MIC of drug A in combination/MIC of drug A alone) + (MIC of drug B in combination/MIC of drug $\mathrm{B}$ alone). FIC indices (FICI) were interpreted as follows: $\leq 0.5$, synergy; $>0.5-\leq 1.0$, additive; $>1.0-\leq 2.0$, 
indifference; and >2.0, antagonism [20].

\subsection{Time-Kill Curves}

The bactericidal activities of the drugs evaluated in this study were also evaluated using time-kill curves constructed using the isolated and reference strains. Cultures with an initial cell density of $1 \times 10^{6}-5 \times 10^{6} \mathrm{CFU} / \mathrm{ml}$ were exposed to the MIC of acacetin alone, or acacetin (1/2 MIC) plus oxacillin (1/2 MIC) or acacetin (1/2 MIC) plus ampicillin (1/2 MIC). Viable counts were conducted at $0,0.5,1,2,3,4,5,6,12$, and $24 \mathrm{~h}$ by plating aliquots of the samples on agar and subsequent incubation for 24 hours at $37^{\circ} \mathrm{C}$. All experiments were repeated several times and colony counts were conducted in duplicate, after which the means were determined.

\section{Results and Discussion}

Many researchers are studying natural products that could be used as antibiotics against MRSA, and are employing novel dosing regimens and antimicrobials that would be advantageous for combating the therapeutic problems associated with S. aureus [9] [10] [21]-[23]. The results of the antibacterial activity showed that the acacetin exhibited inhibitory activities against isolates MRSA and reference stains, MRSA ATCC33591 and MSSA ATCC25923. In Table 1, the acacetin displayed varying degrees of activity against clinical isolated MRSA $1-20$ with MIC in the range of $10-80 \mu \mathrm{g} / \mathrm{mL}$ and MBC in the range of $20-160 \mu \mathrm{g} / \mathrm{mL}$. The MICs/MBCs for ampicillin were determined to be either $64 / 128$ or $1024 / 2048 \mu \mathrm{g} / \mathrm{mL}$; for oxacillin, either $4 / 16$ or 32/64 $\mu \mathrm{g} / \mathrm{mL}$ against MRSA 1 - 20 isolates. The range of MIC $_{50}$ and $\mathrm{MIC}_{90}$ were $1.25-20 \mu \mathrm{g} / \mathrm{mL}$ and $10-80$ $\mu \mathrm{g} / \mathrm{mL}$ against MRSA 1 - 20 isolates, respectively. Flavonoid compounds constitute an important class of phytochemicals which possess diverse biological activities against MRSA [9] [19] [23] [24]. Some of these compounds, like polyphenols, have been shown to exert their antibacterial action through membrane perturbations [25] [26]. The acacetin is known to contain a number of antimicrobial compounds, such as polyphenols and flavonoids. The acacetin, one of main compounds of A. afra showed good inhibitory effects against Gram positive oral bacteria [16].

Table 1. Antibacterial activity of acacetin and antibiotics in isolated MRSA and some of reference bacteria.

\begin{tabular}{|c|c|c|c|c|c|}
\hline \multirow{2}{*}{ Samples } & \multicolumn{3}{|c|}{ Acacetin $(\mu \mathrm{g} / \mathrm{mL})$} & Ampicillin & Oxacillin \\
\hline & $\mathrm{MIC}_{50<}$ & $\mathrm{MIC}_{90<}$ & MIC/MBC & \multicolumn{2}{|c|}{ MIC/MBC $(\mu \mathrm{g} / \mathrm{mL})$} \\
\hline MSSA ATCC $25923^{1}$ & 1.25 & 5 & $5 / 20$ & $8 / 16$ & $0.25 / 1$ \\
\hline MRSA ATCC $33591^{2}$ & 5 & 20 & $20 / 40$ & $1024 / 2048$ & $8 / 16$ \\
\hline MRSA $1^{3}$ & 2.5 & 10 & $10 / 40$ & $1024 / 2048$ & $16 / 32$ \\
\hline MRSA 2 & 5 & 20 & $20 / 80$ & $128 / 256$ & $8 / 16$ \\
\hline MRSA 3 & 10 & 40 & $40 / 80$ & $1024 / 2048$ & $8 / 16$ \\
\hline MRSA 4 & 5 & 20 & $20 / 80$ & 256/512 & $16 / 32$ \\
\hline MRSA 5 & 10 & 40 & $40 / 160$ & $128 / 256$ & $16 / 32$ \\
\hline MRSA 6 & 20 & 80 & $80 / 160$ & $256 / 256$ & $8 / 16$ \\
\hline MRSA 7 & 5 & 20 & $20 / 40$ & $128 / 512$ & $16 / 32$ \\
\hline MRSA 8 & 2.5 & 10 & $10 / 20$ & $128 / 256$ & $8 / 32$ \\
\hline MRSA 9 & 1.25 & 10 & $10 / 40$ & $128 / 512$ & $16 / 32$ \\
\hline MRSA 10 & 10 & 40 & $40 / 80$ & $64 / 128$ & $8 / 16$ \\
\hline MRSA 11 & 5 & 20 & $20 / 80$ & $128 / 256$ & $16 / 64$ \\
\hline MRSA 12 & 10 & 80 & $80 / 160$ & $256 / 256$ & $32 / 64$ \\
\hline MRSA 13 & 20 & 80 & $80 / 160$ & $64 / 128$ & $32 / 64$ \\
\hline MRSA 14 & 5 & 40 & $40 / 80$ & $128 / 256$ & $16 / 32$ \\
\hline MRSA 15 & 10 & 40 & $40 / 160$ & $64 / 128$ & $8 / 16$ \\
\hline MRSA 16 & 20 & 80 & $80 / 160$ & $128 / 256$ & $16 / 32$ \\
\hline MRSA 17 & 2.5 & 10 & $10 / 20$ & $128 / 256$ & $8 / 16$ \\
\hline MRSA 18 & 20 & 80 & $80 / 160$ & $64 / 128$ & $8 / 16$ \\
\hline MRSA 19 & 20 & 80 & $80 / 160$ & $64 / 128$ & $4 / 16$ \\
\hline MRSA 20 & 5 & 40 & $40 / 80$ & $128 / 512$ & $16 / 32$ \\
\hline
\end{tabular}

${ }^{1}$ MSSA (ATCC 25923): reference strain Methicillin-sensitive Staphylococcus aureus; ${ }^{2}$ MRSA (ATCC 33591): reference strain Methicillin-resistant Staphylococcus aureus; ${ }^{3}$ MRSA (1 - 20): Methicillin-resistant Staphylococcus aureus isolated a clinic. 
Combination antibiotic therapy has been studied to promote the effective use of antibiotics in increasing in vivo activity of antibiotics, in preventing the spread of drug-resistant strains, and in minimizing toxicity [7] [17] [20]. The combination of oxacillin and acacetin resulted in a reduction in the MICs/MBCs for all bacteria, with the MICs/MBCs of $1.25 / 5$ or $20 / 80 \mu \mathrm{g} / \mathrm{mL}$ for oxacillin becoming $0.0625-8 / 0.25-16 \mu \mathrm{g} / \mathrm{mL}$ and reduced by $\geq 4$-fold in most of $S$. aureus tested, evidencing a synergistic effect as defined by a FICI of $\leq 0.5$ except clinic MRSA 3, 8, 9, and 10 at MIC and clinic MRSA 3, 5, 6, 10, 13, 15, 17, and 20 at MBC (Table 2). In

Table 2. Synergistic effects of the acacetin with oxacillin in isolated MRSA and some of reference bacteria.

\begin{tabular}{|c|c|c|c|c|c|c|}
\hline \multirow{2}{*}{ Samples } & \multirow{2}{*}{ Agent } & \multicolumn{2}{|c|}{$\mathrm{MIC} / \mathrm{MBC}(\mu \mathrm{g} / \mathrm{mL})$} & \multirow{2}{*}{ FIC/FBC } & \multirow{2}{*}{$\mathrm{FICI} / \mathrm{FBCI}^{2}$} & \multirow{2}{*}{ Outcome } \\
\hline & & Alone & Combination $^{1}$ & & & \\
\hline \multirow{2}{*}{ MSSA ATCC $25923^{3}$} & Acacetin & $5 / 20$ & $1.25 / 5$ & $0.25 / 0.25$ & \multirow{2}{*}{$0.5 / 0.5$} & \multirow{2}{*}{ Synergistic/Synergistic } \\
\hline & Oxacillin & $0.25 / 1$ & $0.0625 / 0.25$ & $0.25 / 0.25$ & & \\
\hline \multirow{2}{*}{ MRSA ATCC $33591^{4}$} & Acacetin & $20 / 40$ & $5 / 10$ & $0.25 / 0.25$ & \multirow{2}{*}{$0.5 / 0.5$} & \multirow{2}{*}{ Synergistic/Synergistic } \\
\hline & Oxacillin & $8 / 16$ & $2 / 4$ & $0.25 / 0.25$ & & \\
\hline \multirow{2}{*}{ MRSA $1^{5}$} & Acacetin & $10 / 40$ & $2.5 / 10$ & $0.25 / 0.25$ & \multirow{2}{*}{$0.5 / 0.5$} & \multirow{2}{*}{ Synergistic/Synergistic } \\
\hline & Oxacillin & $16 / 32$ & $4 / 8$ & $0.25 / 0.25$ & & \\
\hline \multirow{2}{*}{ MRSA 2} & Acacetin & $20 / 80$ & $5 / 10$ & $0.25 / 0.125$ & \multirow{2}{*}{$0.5 / 0.375$} & \multirow{2}{*}{ Synergistic/Synergistic } \\
\hline & Oxacillin & $8 / 16$ & $2 / 4$ & $0.25 / 0.25$ & & \\
\hline \multirow{2}{*}{ MRSA 3} & Acacetin & $40 / 80$ & $10 / 20$ & $0.25 / 0.25$ & \multirow{2}{*}{$0.75 / 0.75$} & \multirow{2}{*}{ Additive/Additive } \\
\hline & Oxacillin & $8 / 16$ & $4 / 8$ & $0.5 / 0.5$ & & \\
\hline \multirow{2}{*}{ MRSA 4} & Acacetin & $20 / 80$ & $5 / 10$ & $0.25 / 0.125$ & \multirow{2}{*}{$0.5 / 0.375$} & Symoraictic/Additive \\
\hline & Oxacillin & $16 / 32$ & $4 / 8$ & $0.25 / 0.25$ & & synergisuct Aunatuve \\
\hline MPSA 5 & Acacetin & $40 / 160$ & $10 / 40$ & $0.25 / 0.25$ & $05 / 075$ & Sumoraictic/Additive \\
\hline VIRSA & Oxacillin & $16 / 32$ & $4 / 16$ & $0.25 / 0.5$ & $0.2 / 0 . / 5$ & synergisuc/ Aunutuve \\
\hline & Acacetin & $80 / 160$ & $20 / 40$ & $0.25 / 0.25$ & $05 / 075$ & Syneroistic/Additive \\
\hline MRSA 6 & Oxacillin & $8 / 16$ & $2 / 8$ & $0.25 / 0.5$ & $0.5 / 0.75$ & synergistic/Additıve \\
\hline MRSA 7 & Acacetin & $20 / 40$ & $5 / 10$ & $0.25 / 0.25$ & $05 / 05$ & Symerojistic/Synoroistic \\
\hline MRSA I & Oxacillin & $16 / 32$ & $4 / 8$ & $0.25 / 0.25$ & 0.5/0.5 & synergistic/synergistic \\
\hline MPSA 9 & Acacetin & $10 / 20$ & $5 / 10$ & $0.5 / 0.5$ & $075 / 0375$ & Additivo/Sunorgictic \\
\hline MRSA 8 & Oxacillin & $8 / 32$ & $2 / 4$ & $0.25 / 0.125$ & $0 . / 5 / 0.3 / 5$ & Additıve/synergistic \\
\hline MRSA 9 & Acacetin & $10 / 40$ & $2.5 / 5$ & $0.25 / 0.125$ & $075 / 0375$ & Additive/Syneroistic \\
\hline & Oxacillin & $16 / 32$ & $8 / 8$ & $0.5 / 0.25$ & $0 . / 5 / 0.3 / 5$ & Additıve/synergistıc \\
\hline MRSA 10 & Acacetin & $40 / 80$ & $10 / 20$ & $0.25 / 0.25$ & $075 / 075$ & Syneroistic/Synoroistic \\
\hline & Oxacillin & $8 / 16$ & $4 / 8$ & $0.5 / 0.5$ & $0.15 / 0 . / 5$ & synergistic/s syitergistic \\
\hline MRSA 11 & Acacetin & $20 / 80$ & $5 / 20$ & $0.25 / 0.25$ & $0.5 / 0.375$ & Synergistic/Synergistic \\
\hline МИКО II & Oxacillin & $16 / 64$ & $4 / 8$ & $0.25 / 0.125$ & $0.3 / 0.3 / 5$ & symergistic/synergistic \\
\hline MRSA 12 & Acacetin & $80 / 160$ & $20 / 40$ & $0.25 / 0.25$ & $0.375 / 0.5$ & Synergistic/Synergistic \\
\hline MRSA 12 & Oxacillin & $32 / 64$ & $4 / 16$ & $0.125 / 0.25$ & 0.3/5/0.5 & Synergistic/synergistic \\
\hline MRSA 13 & Acacetin & $80 / 160$ & $20 / 80$ & $0.25 / 0.5$ & $0.375 / 0.75$ & Synergistic/Additive \\
\hline 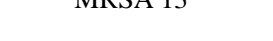 & Oxacillin & $32 / 64$ & $4 / 16$ & $0.125 / 0.25$ & & \\
\hline MRSA 14 & Acacetin & $40 / 80$ & $10 / 20$ & $0.25 / 0.25$ & $05 / 05$ & Syneroistic/Syneroistic \\
\hline & Oxacillin & $16 / 32$ & $4 / 8$ & $0.25 / 0.25$ & $0.5 / 0.5$ & synergistuc/ syntergistic \\
\hline MRSA 15 & Acacetin & $40 / 160$ & $10 / 40$ & $0.25 / 0.25$ & $0.5 / 0.75$ & Synergistic/Additive \\
\hline & Oxacillin & $8 / 16$ & $2 / 8$ & $0.25 / 0.5$ & & \\
\hline MRSA 16 & Acacetin & $80 / 160$ & $20 / 40$ & $0.25 / 0.25$ & $05 / 05$ & Symorojistic/Symoroistic \\
\hline MIRSA LO & Oxacillin & $16 / 32$ & $4 / 8$ & $0.25 / 0.25$ & $0.5 / 0.5$ & symergistic/synergistic \\
\hline MRSA 17 & Acacetin & $10 / 20$ & $2.5 / 10$ & $0.25 / 0.5$ & $0.5 / 10$ & Synergistic/Additive \\
\hline & Oxacillin & $8 / 16$ & $2 / 8$ & $0.25 / 0.5$ & & \\
\hline MRSA 18 & Acacetin & $80 / 160$ & $20 / 40$ & $0.25 / 0.25$ & $05 / 05$ & Syneroistic/Syneroistic \\
\hline NMKSA 10 & Oxacillin & $8 / 16$ & $2 / 4$ & $0.25 / 0.25$ & & 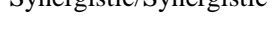 \\
\hline MRSA 19 & Acacetin & $80 / 160$ & $20 / 40$ & $0.25 / 0.25$ & $0.5 / 0.375$ & Synergistic/Synergistic \\
\hline MRSA 19 & Oxacillin & $4 / 16$ & $1 / 2$ & $0.25 / 0.125$ & $0.3 / 0.3 / 5$ & symergistic/synergistic \\
\hline MRSA 20 & Acacetin & $40 / 80$ & $10 / 20$ & $0.25 / 0.25$ & $0.5 / 0.75$ & Synergistic/Additive \\
\hline NIKSA $\angle U$ & Oxacillin & $16 / 32$ & $4 / 16$ & $0.25 / 0.5$ & $0.3 / 0.15$ & synergisuc/ Aucumve \\
\hline
\end{tabular}

${ }^{1}$ The MIC and MBC of acacetin with oxacillin; ${ }^{2}$ The FIC index; ${ }^{3}$ MSSA (ATCC 25923): reference strain Methicillin-sensitive Staphylococcus aureus; ${ }^{4}$ MRSA (ATCC 33591): reference strain Methicillin-resistant Staphylococcus aureus; ${ }^{5}$ MRSA (1 - 20): Methicillin-resistant Staphylococcus aureus isolated a clinic. 
combination with acacetin, the MICs/MBCs for ampicillin were reduced by $\geq 4$-fold in most of $S$. aureus tested, evidencing a synergistic effect as defined by a FICI of $\leq 0.5$ except clinic MRSA 7, 10, 11, 16, and 19 at MIC and clinic MRSA 6, 8, 10, 11, 12, 13, 15, and 19 at MBC by FICI of $>0.625$ (Table 3). The effects of acacetin

Table 3. Synergistic effects of acacetin with ampicillin in isolated MRSA and some of reference bacteria.

\begin{tabular}{|c|c|c|c|c|c|c|}
\hline \multirow{2}{*}{ Samples } & \multirow{2}{*}{ Agent } & \multicolumn{2}{|c|}{ MIC/MBC $(\mu \mathrm{g} / \mathrm{mL})$} & \multirow{2}{*}{ FIC/FBC } & \multirow{2}{*}{$\mathrm{FICI} / \mathrm{FBCI}^{2}$} & \multirow{2}{*}{ Outcome } \\
\hline & & Alone & Combination $^{1}$ & & & \\
\hline \multirow{2}{*}{ MSSA ATCC $25923^{3}$} & Acacetin & $5 / 20$ & $1.25 / 5$ & $0.25 / 0.25$ & \multirow{2}{*}{$0.5 / 0.5$} & \multirow{2}{*}{ Synergistic/Synergistic } \\
\hline & Ampicillin & $8 / 16$ & $2 / 4$ & $0.25 / 0.25$ & & \\
\hline \multirow{2}{*}{ MRSA ATCC $33591^{4}$} & Acacetin & $20 / 40$ & $5 / 10$ & $0.25 / 0.25$ & \multirow{2}{*}{$0.5 / 0.5$} & \multirow{2}{*}{ Synergistic/Synergistic } \\
\hline & Ampicillin & $1024 / 2048$ & $256 / 512$ & $0.25 / 0.25$ & & \\
\hline \multirow{2}{*}{ MRSA $1^{5}$} & Acacetin & $10 / 40$ & $2.5 / 10$ & $0.25 / 0.25$ & \multirow{2}{*}{$0.5 / 0.5$} & \multirow{2}{*}{ Synergistic/Synergistic } \\
\hline & Ampicillin & $1024 / 2048$ & $256 / 512$ & $0.25 / 0.25$ & & \\
\hline \multirow{2}{*}{ MRSA 2} & Acacetin & $20 / 80$ & $5 / 20$ & $0.25 / 0.25$ & \multirow{2}{*}{$0.5 / 0.5$} & \multirow{2}{*}{ Synergistic/Synergistic } \\
\hline & Ampicillin & $128 / 256$ & $32 / 64$ & $0.25 / 0.25$ & & \\
\hline \multirow{2}{*}{ MRSA 3} & Acacetin & $40 / 80$ & $10 / 20$ & $0.25 / 0.25$ & \multirow{2}{*}{$0.5 / 0.5$} & \multirow{2}{*}{ Synergistic/Synergistic } \\
\hline & Ampicillin & $1024 / 2048$ & $256 / 512$ & $0.25 / 0.25$ & & \\
\hline \multirow{2}{*}{ MRSA 4} & Acacetin & $20 / 80$ & $5 / 10$ & $0.25 / 0.125$ & \multirow{2}{*}{$0.5 / 0.375$} & Synergistic/Synergistic \\
\hline & Ampicillin & $256 / 512$ & $64 / 128$ & $0.25 / 0.25$ & & syliergisuc/syntergisuc \\
\hline & Acacetin & $40 / 160$ & $10 / 40$ & $0.25 / 0.25$ & & \\
\hline MRSA S & Ampicillin & $128 / 256$ & $32 / 64$ & $0.25 / 0.25$ & $0.5 / 0.5$ & Synergistic/Synergistic \\
\hline MRSA 6 & Acacetin & $80 / 160$ & $20 / 40$ & $0.25 / 0.25$ & $0.5 / 075$ & \\
\hline MINSA O & Ampicillin & $256 / 256$ & $64 / 128$ & $0.25 / 0.5$ & $0.5 / 0.75$ & Synergistic/Additive \\
\hline MRSA 7 & Acacetin & $20 / 40$ & $5 / 10$ & $0.25 / 0.25$ & $075 / 0.5$ & Additive/Syneroistic \\
\hline MIRSA I & Ampicillin & $128 / 512$ & $64 / 128$ & $0.5 / 0.25$ & $0 . / 5 / 0.5$ & Adantive/synergistic \\
\hline MPSA 8 & Acacetin & $10 / 20$ & $2.5 / 10$ & $0.25 / 0.5$ & $0.5 / 0.75$ & Synorrictic/Additiva \\
\hline МIRSA O & Ampicillin & $128 / 256$ & $32 / 64$ & $0.25 / 0.25$ & 0.J/0./J & synergisuc/Auduve \\
\hline MRSA 9 & Acacetin & $10 / 40$ & $2.5 / 10$ & $0.25 / 0.25$ & $0.5 / 0375$ & Syneroistic/Synergistic \\
\hline IMRSA Y & Ampicillin & $128 / 512$ & $32 / 64$ & $0.25 / 0.125$ & $0.5 / 0.3 / 5$ & synergisuc/synergisuc \\
\hline MRSA 10 & Acacetin & $40 / 80$ & $10 / 20$ & $0.25 / 0.25$ & $075 / 075$ & Additive/Additive \\
\hline MISAA 10 & Ampicillin & $64 / 128$ & $32 / 64$ & $0.5 / 0.5$ & $0.15 / 0.15$ & Adartive/Adantive \\
\hline MRSA 11 & Acacetin & $20 / 80$ & $5 / 10$ & $0.25 / 0.125$ & $075 / 0.625$ & Additive/Additive \\
\hline MVKSA II & Ampicillin & $128 / 256$ & $64 / 128$ & $0.5 / 0.5$ & $0.15 / 0.025$ & Aduitive/Auditive \\
\hline MRSA 12 & Acacetin & $80 / 160$ & $20 / 40$ & $0.25 / 0.25$ & $0.5 / 0.75$ & Synergistic/Additive \\
\hline IVIRSA 12 & Ampicillin & $256 / 256$ & $64 / 128$ & $0.25 / 0.5$ & 0.J/0./J & synergisuc/Auduve \\
\hline MRSA 13 & Acacetin & $80 / 160$ & $20 / 40$ & $0.25 / 0.25$ & $0.5 / 0.75$ & Synergistic/Additive \\
\hline & Ampicillin & $64 / 128$ & $16 / 64$ & $0.25 / 0.5$ & $0.3 / 0.15$ & synergisuc/Auduve \\
\hline MRSA 14 & Acacetin & $40 / 80$ & $10 / 20$ & $0.25 / 0.25$ & $0.5 / 0.5$ & Synergistic/Synergistic \\
\hline & Ampicillin & $128 / 256$ & $32 / 64$ & $0.25 / 0.25$ & & \\
\hline MRSA 15 & Acacetin & $40 / 160$ & $10 / 40$ & $0.25 / 0.25$ & $0.5 / 075$ & Syneroistic/Additive \\
\hline IVINSA IS & Ampicillin & $64 / 128$ & $16 / 64$ & $0.25 / 0.5$ & $0.0 / 0.13$ & 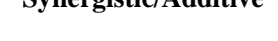 \\
\hline MRSA 16 & Acacetin & $80 / 160$ & $20 / 40$ & $0.25 / 0.25$ & $075 / 0.5$ & Additive/Syneroistic \\
\hline 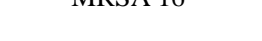 & Ampicillin & $128 / 256$ & $64 / 64$ & $0.5 / 0.25$ & 0.7010 .0 & 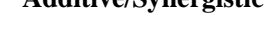 \\
\hline MRSA 17 & Acacetin & $10 / 20$ & $2.5 / 5$ & $0.25 / 0.25$ & $05 / 05$ & Synergistic/Synergistic \\
\hline IVIRSA 17 & Ampicillin & $128 / 256$ & $32 / 64$ & $0.25 / 0.25$ & $0.3 / 0.3$ & synergisuc/synergisuc \\
\hline MRSA 18 & Acacetin & $80 / 160$ & $20 / 40$ & $0.25 / 0.25$ & $0.5 / 0.5$ & Synergistic/Synergistic \\
\hline ИVКОА 10 & Ampicillin & $64 / 128$ & $16 / 32$ & $0.25 / 0.25$ & $0.5 / 0.5$ & synergistic/synergistic \\
\hline MRSA 19 & Acacetin & $80 / 160$ & $20 / 40$ & $0.25 / 0.25$ & $075 / 075$ & Additive/Additive \\
\hline ПИКОА & Ampicillin & $64 / 128$ & $32 / 64$ & $0.5 / 0.5$ & $0 . / 3 / 0 . / 3$ & Adumive/Auditive \\
\hline MRSA 20 & Acacetin & $40 / 80$ & $10 / 20$ & $0.25 / 0.25$ & $05 / 0375$ & Synergistic/Synergistic \\
\hline & Ampicillin & $128 / 512$ & $32 / 64$ & $0.25 / 0.125$ & & symergisuc/ \\
\hline
\end{tabular}

${ }^{1}$ The MIC and MBC of acacetin with ampicillin; ${ }^{2}$ The FIC index; ${ }^{3}$ MSSA (ATCC 25923): reference strain Methicillin-sensitive Staphylococcus aureus; ${ }^{4}$ MRSA (ATCC 33591): reference strain Methicillin-resistant Staphylococcus aureus; ${ }^{5}$ MRSA (1 - 20): Methicillin-resistant Staphylococcus aureus isolated a clinic. 
administered in combination with oxacillin or ampicillin against standard (MSSA and MRSA) and clinical isolates of MRSA (MRSA 1 - 20) were confirmed by time-kill curve experiments (Figures 1-4). Cultures of each strain of bacteria with a cell density of $10^{6} \mathrm{CFU} / \mathrm{mL}$ were exposed to the MIC of acacetin alone or acacetin $(1 / 2$ MIC) with oxacillin (1/2 MIC) or ampicillin (1/2 MIC). We observed that 30 minutes of acacetin treatment with ampicillin or oxacillin resulted in a rapidly increased rate of killing as compared to that observed with acacetin (MIC) alone (Figures 1-4).
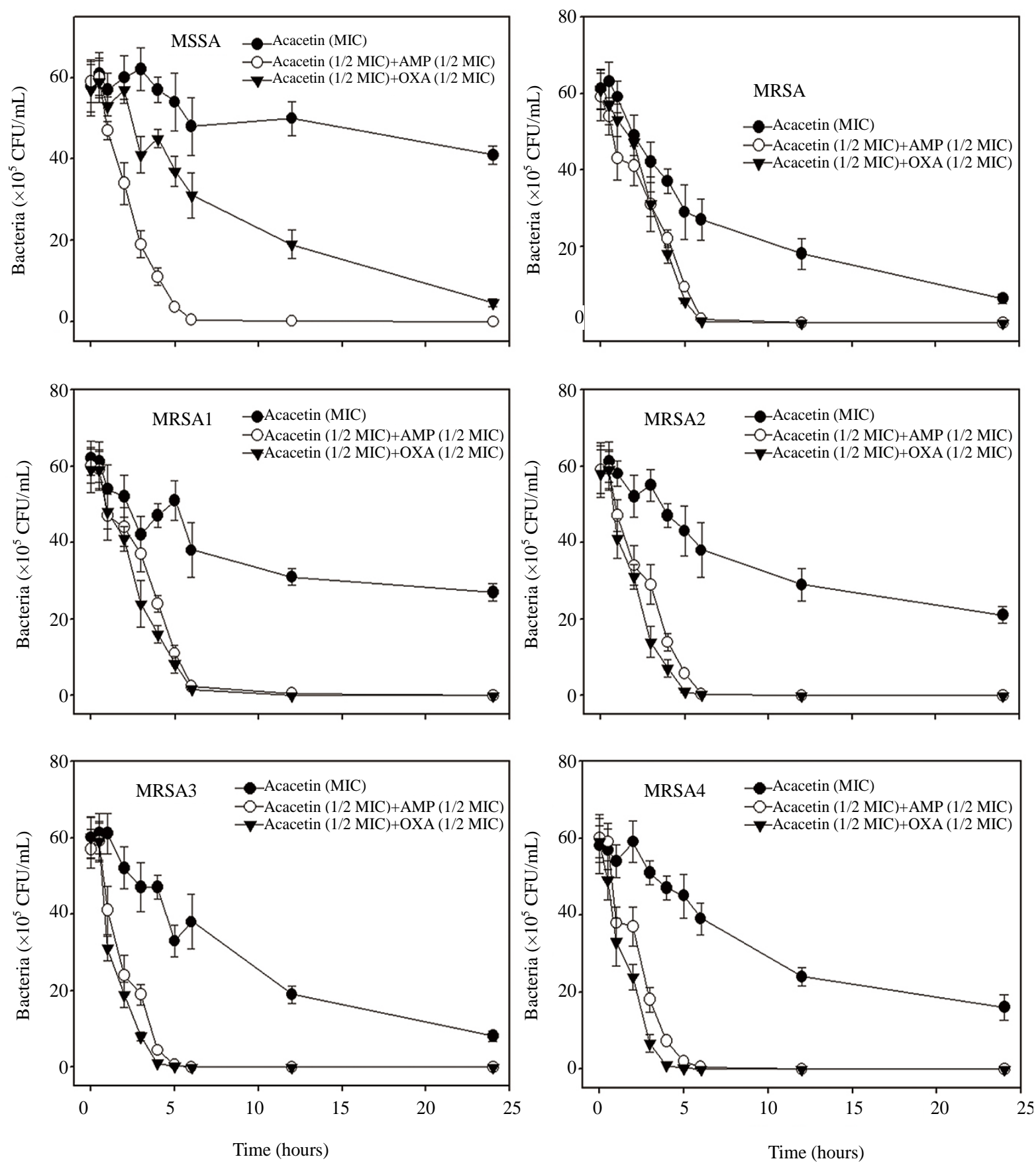

Figure 1. Time-kill curves of MIC of the acacetin alone and 1/2 MIC of acacetin with 1/2 MIC of oxacillin or ampicillin against isolates MRSA (1-4) and methicillin-sensitive S. aureus (MSSA) ATCC 25923 and methicillin-resistant S. aureus (MRSA) ATCC 33591 strains. Bacteria were incubated with the acacetin alone $(\bullet)$ and with ampicillin $(\circ)$ or with oxacillin ( $\boldsymbol{\nabla})$ over time. CFU, colony-forming units. 

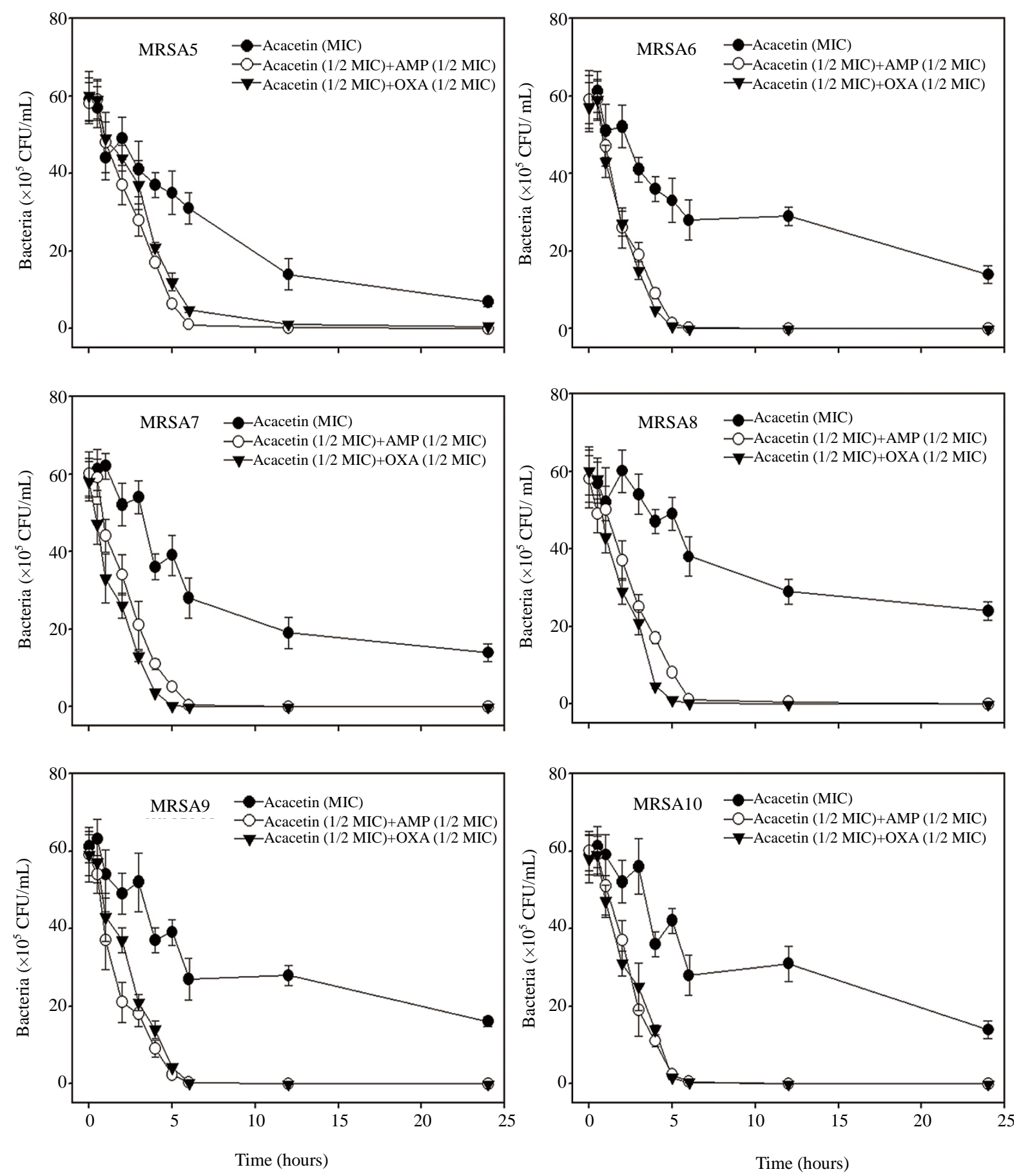

Figure 2. Time-kill curves of MIC of the acacetin alone and 1/2 MIC of acacetin with 1/2 MIC of oxacillin or ampicillin against isolates MRSA (5 - 10). Bacteria were incubated with the acacetin alone $(\bullet)$ and with ampicillin $(0)$ or with oxacillin $(\boldsymbol{\nabla})$ over time. CFU, colony-forming units.

A profound bactericidal effect was exerted when a combination of drugs was utilized. The growth of the tested bacteria was completely attenuated after $2-6 \mathrm{~h}$ of treatment with the 1/2 MIC of acacetin, regardless of whether it was administered alone or with oxacillin (1/2 MIC) or ampicillin (1/2 MIC) except MRSA 15 and 17. Flavonoids affect bacterial membrane potential and cause permeability alteration within the inner microorganisms membrane [27]-[29]. This perturbation of the cell membrane coupled with the action of $\beta$-lactams on 

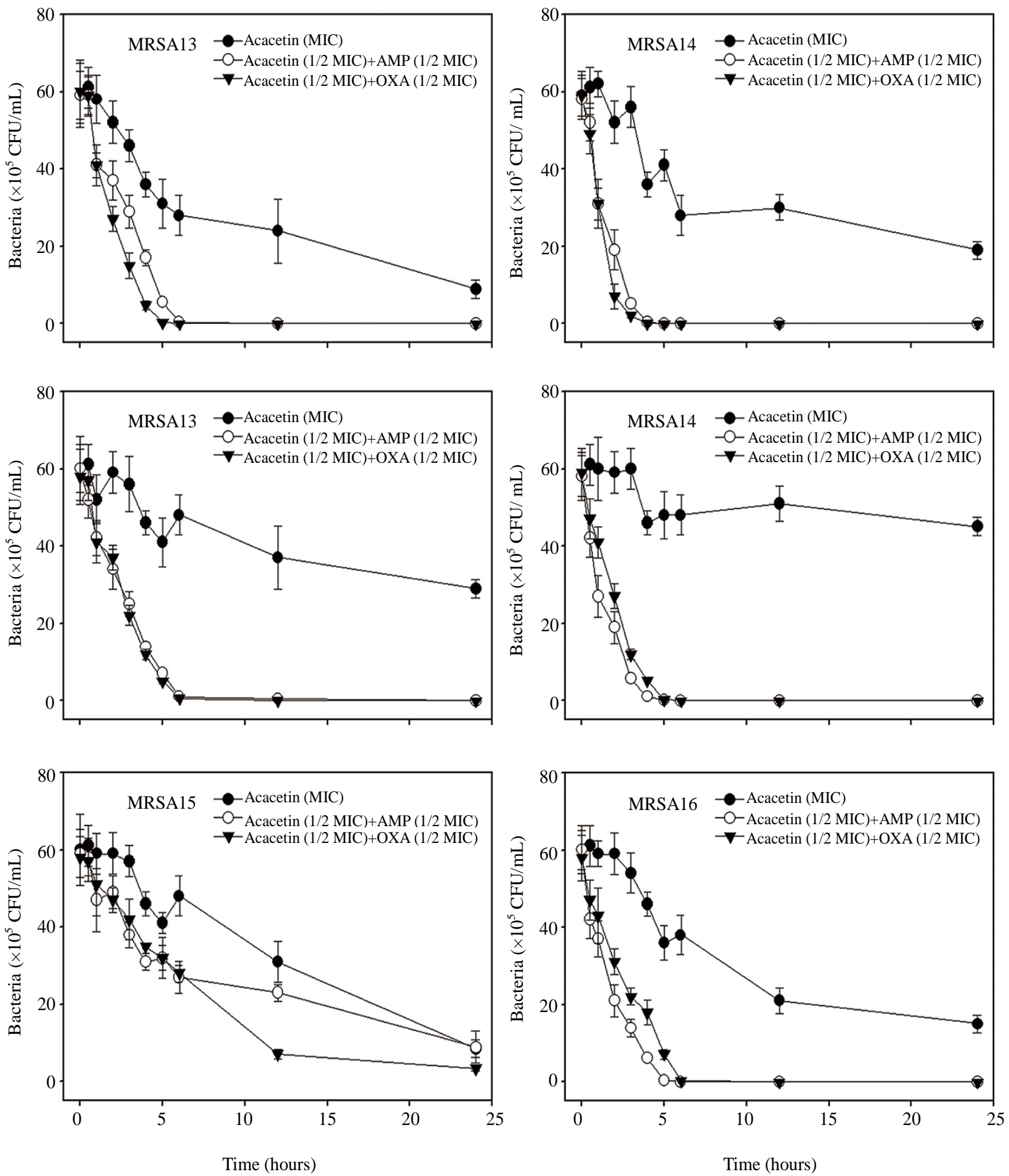

Figure 3. Time-kill curves of MIC of the acacetin alone and 1/2 MIC of acacetin with 1/2 MIC of oxacillin or ampicillin against isolates MRSA (11 - 16). Bacteria were incubated with the acacetin alone $(\bullet)$ and with ampicillin $(\circ)$ or with oxacillin $(\boldsymbol{\nabla})$ over time. CFU, colony-forming units.

the transpeptidation of the cell membrane could lead to the enhanced antimicrobial effect [27] [29].

In conclusion, acacetin exerted synergistic effects when administered with oxacillin or ampicillin and the antimicrobial effect and resistant regulation of acacetin against MRSA might be useful for potential application as a natural product agent. 

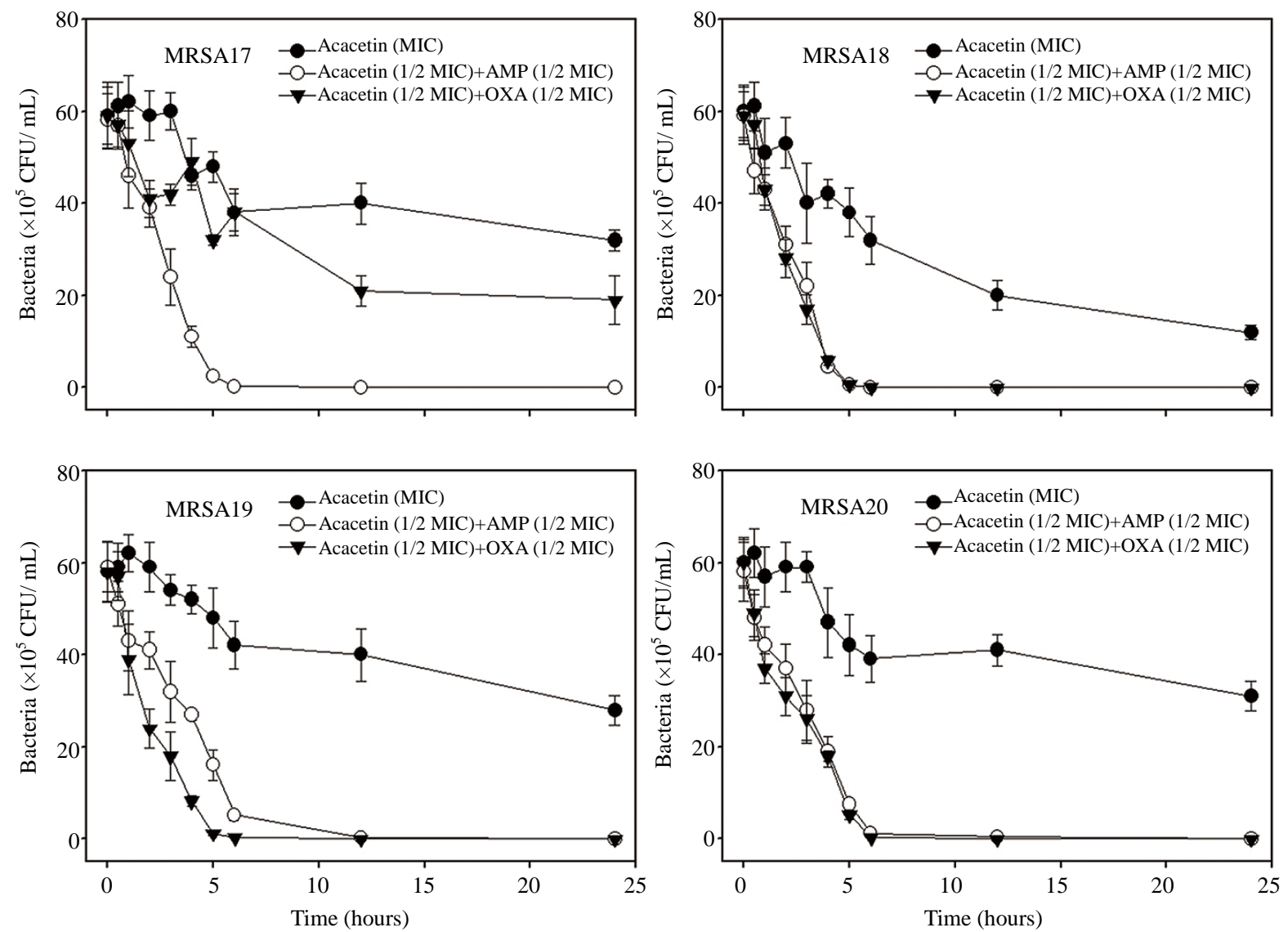

Figure 4. Time-kill curves of MIC of the acacetin alone and 1/2 MIC of acacetin with 1/2 MIC of oxacillin or ampicillin against isolates MRSA (17 - 20). Bacteria were incubated with the acacetin alone $(\bullet)$ and with ampicillin (०) or with oxacillin $(\boldsymbol{\nabla})$ over time. CFU, colony-forming units.

\section{Acknowledgements}

This research was supported by Dong-eui University Grant (2011AA106). There is no conflict of interest related to this research.

\section{References}

[1] Stefani, S. and Varaldo, P.E. (2003) Epidemiology of Methicillin Resistant Staphylococci in Europe. Clinical Microbiology and Infection, 9, 1179-1186. http://dx.doi.org/10.1111/j.1469-0691.2003.00698.x

[2] Pourakbari, B., Rezaizadeh, G., Mahmoudi, S. and Mamishi, S. (2012) Epidemiology of Nosocomial Infections in Pediatric Patients in an Iranian Referral Hospital. Journal of Preventive Medicine and Hygiene, 53, 204-206.

[3] Krishna, S. and Miller, L.S. (2012) Host-Pathogen Interactions between the Skin and Staphylococcus aureus. Current Opinion in Microbiology, 15, 28-35. http://dx.doi.org/10.1016/j.mib.2011.11.003

[4] Cleven, B.E., Palka-Santini, M., Gielen, J., Meembor, S., Kronke, M. and Krut, O. (2006) Identification and Characterization of Bacterial Pathogens Causing Bloodstream Infections by DNA Microarray. Journal of Clinical Microbiology, 44, 2389-2397. http://dx.doi.org/10.1128/JCM.02291-05

[5] Zecconi, A. and Scali, F. (2013) Staphylococcus aureus Virulence Factors in Evasion from Innate Immune Defenses in Human and Animal Diseases. Immunology Letters, 150, 12-22. http://dx.doi.org/10.1016/j.imlet.2013.01.004

[6] Thati, V., Shivannavar, C.T. and Gaddad, S.M. (2011) Vancomycin Resistance among Methicillin Resistant Staphylococcus aureus Isolates from Intensive Care Units of Tertiary Care Hospitals in Hyderabad. The Indian Journal of Medical Research, 134, 704-708. http://dx.doi.org/10.4103/0971-5916.91001

[7] Périchon, B. and Courvalin, P. (2006) Synersism between Beta-Lactams and Glycopeptides against VanA-Type Methicillin-Resistant Staphylococcus aureus and Heterologous Expression of the vanA Operon. Antimicrobial Agents and 
Chemotherapy, 50, 3622-3630. http://dx.doi.org/10.1128/AAC.00410-06

[8] Dahiya, P. and Purkayastha, S. (2012) Phytochemica Screening and Antimicrobial Activity of Some Medicinal Plant Multi-Drug Resistant Bacteria from Clinical Isolates. Indian Journal of Pharmaceutical Sciences, 74, 443-450. http://dx.doi.org/10.4103/0250-474X.108420

[9] Su, X., Howell, A.B. and D’Souza, D.H. (2012) Antibacterial Effects of Plant-Derived Extracts on Methicillin-Resistant Staphylococcus aureus. Foodborne Pathogens and Disease, 9, 573-578. http://dx.doi.org/10.1089/fpd.2011.1046

[10] Sasaki, H., Kashiwada, Y., Shibata, H. and Takaishi, Y. (2012) Prenylated Flavonoids from Desmodium caudatum and Evaluation of Their Anti-MRSA Activity. Phytochemistry, 82, 136-142. http://dx.doi.org/10.1016/j.phytochem.2012.06.007

[11] Rasul, A., Millimouno, F.M., Ali Eltayb, W., Ali, M., Li, J. and Li, X. (2013) Pinocembrin: A Novel Natural Compound with Versatile Pharmacological and Biological Activities. BioMed Research International, 2013, Article ID: 379850. http://dx.doi.org/10.1155/2013/379850

[12] Chirumbolo, S. (2010) The Role of Quercetin, Flavonols and Flavones in Modulating Inflammatory Cell Function. Inflammation Allergy Drug Targets, 9, 263-285.

[13] Czaplińska, M., Czepas, J. and Gwoździński, K. (2012) Structure, Antioxidative and Anticancer Properties of Flavonoids. Postepy Biochemii, 58, 235-244.

[14] Fong, Y., Shen, K.H., Chiang, T.A. and Shih, Y.W. (2010) Acacetin Inhibits TPA-Induced MMP-2 and u-PA Expressions of Human Lung Cancer Cells through Inactivating JNK Signaling Pathway and Reducing Binding Activities of NF-kappaB and AP-1. Journal of Food Science, 75, 1750-3841. http://dx.doi.org/10.1111/j.1750-3841.2009.01438.x

[15] Ha, S.K., Moon, E., Lee, P., Ryu, J.H., Oh, M.S. and Kim, S.Y. (2012) Acacetin Attenuates Neuroinflammation via Regulation the Response to LPS Stimuli in Vitro and in Vivo. Neurochemical Research, 37, 1560-1567. http://dx.doi.org/10.1007/s11064-012-0751-z

[16] da Cunha, M.G., Franchin, M., de Carvalho Galvao, L.C., de Ruiz, A.L., de Carvalho, J.E., Ikegaki, M., de Alencar, S.M., Koo, H. and Rosalen, P.L. (2013) Antimicrobial and Antiproliferative Activities of Stingless Bee Melipona scutellaris Geopropolis. BMC Complementary \& Alternative Medicine, 28, 23. http://dx.doi.org/10.1186/1472-6882-13-23

[17] Qin, R., Xiao, K., Li, B., Jiang, W., Peng, W., Zheng, J. and Zhou, H. (2013) The Combination of Catechin and Epicatechin Gallate from Fructus Crataegi Potentiates Beta-Lactam Antibiotics against Methicillin-Resistant Staphylococcus aureus (MRSA) in Vitro and in Vivo. International Journal of Molecular Sciences, 14, 1802-1821. http://dx.doi.org/10.3390/ijms14011802

[18] Wallet, F., Roussel-Delvallez, M. and Courcol, R.J. (1996) Choice of a Routine Method for Detecting Methicillin-Resistance in Staphylococci. The Journal of Antimicrobial Chemotherapy, 37, 901-909. http://dx.doi.org/10.1093/jac/37.5.901

[19] Cha, J.D., Jeong, M.R., Jeong, S.I. and Lee, K.Y. (2007) Antibacterial Activity of Sophoraflavanone G Isolated from the Roots of Sophora flavescens. Journal of Microbiology and Biotechnology, 17, 858-864.

[20] Climo, M.W., Patron, R.L. and Archer, G.L. (1999) Combinations of Vancomycin and Beta-Lactams Are Synergistic against Staphylococci with Reduced Susceptibilities to Vancomycin. Antimicrobial Agents and Chemotherapy, 43, 1747-1753.

[21] Gibbons, S., Leimkugel, J., Oluwatuyi, M. and Heinrich, M. (2003) Activity of Zanthoxylum clava-herculis Extracts against Multi-Drug Resistant Methicillin-Resistant Staphylococcus aureus (mdr-MRSA). Phytotherapy Research, 17, 274-275. http://dx.doi.org/10.1002/ptr.1112

[22] Kitahara, T., Aoyama, Y., Hirakata, Y., Kamihira, S., Kohno, S., Ichikawa, N., Nakashima, M., Sasaki, H. and Higuchi, S. (2006) In Vitro Activity of Lauric Acid or Myristylamine in Combination with Six Antimicrobial Agents against Methicillin-Resistant Staphylococcus aureus (MRSA). International Journal of Antimicrobial Agents, 27, 51-57. http://dx.doi.org/10.1016/j.ijantimicag.2005.08.020

[23] Sato, Y., Suzakim S., Nishikawa, T., Kihara, M., Shibata, H. and Higuti, T. (2000) Phytochemical Flavones Isolated from Scutellaria barbata and Antibacterial Activity against Methicillin-Resistant Staphylococcus aureus. Journal of Ethnopharmacology, 72, 484-488. http://dx.doi.org/10.1016/S0378-8741(00)00265-8

[24] Cushnie, T.P. and Lamb, A.J. (2005) Antimicrobial Activity of Flavonoids. International Journal of Antimicrobial Agents, 26, 343-356. http://dx.doi.org/10.1016/j.ijantimicag.2005.09.002

[25] Yi, S.M., Zhu, J.L., Fu, L.L. and Li, J.R. (2010) Tea Polyphenols Inhibit Pseudomonas aeruginosa through Damage to the Cell Membrane. International Journal of Food Microbiology, 144, 111-117. http://dx.doi.org/10.1016/j.ijfoodmicro.2010.09.005

[26] Yi, S., Wang, W., Bai, F., Zhu, J., Li, J., Li, X., Xu, Y., Sun, T. and He, Y. (2013) Antimicrobial Effect and Membrane-Active Mechanism of Tea Polyphenols against Serratia marcescens. World Journal of Microbiology \& Biotechnology, 27. 
[27] Holler, J.G., Slotved, H.C., Mølgaard, P., Olsen, C.E. and Christensen, S.B. (2012) Chalcone Inhibitors of the NorA Efflux Pump in Staphylococcus aureus Whole Cells and Enriched Everted Membrane Vesicles. Bioorganic \& Medicinal Chemistry, 20, 4514-4521. http://dx.doi.org/10.1016/j.bmc.2012.05.025

[28] Roselli, M., Britti, M.S., Le Huërou-Luron, I., Marfaing, H., Zhu, W.Y. and Mengheri, E. (2007) Effect of Different Plant Extracts and Natural Substances (PENS) against Membrane Damage Induced by Enterotoxigenic Escherichia coli K88 in Pig Interstinal Cells. Toxicology in Vitro, 21, 224-229. http://dx.doi.org/10.1016/j.tiv.2006.09.012

[29] Liu, X.L., Zhang, X.J., Fu, Y.J., Zu, Y.G., Wu, N., Liang, L. and Efferth, T. (2011) Cajanol Inhibits the Growth of Escherichia coli and Staphylococcus aureus by Acting on Membrane and DNA Damage. Planta Medica, 77, 158-163. http://dx.doi.org/10.1055/s-0030-1250146 